\title{
Lattice Investigations of the Hosotani Mechanism of Spontaneous Symmetry Breaking
}

\author{
James E. Hetrick* \\ Physics Department \\ University of the Pacific Stockton, CA 95211 USA \\ E-mail: jhetrick@pacific.edu
}

\begin{abstract}
The Hosotani Mechanism is a method of dynamical gauge symmetry breaking which can occur in gauge theories with compact extra dimensions. Under certain conditions (fermion content and boundary conditions) the gauge field component in the compact dimension develops a VEV that is outside the center of the gauge group. Investigations of this phenomenon in $3+1$ dimensions are reported, particularly the behavior of gauge correlators.
\end{abstract}

31st International Symposium on Lattice Field Theory - LATTICE 2013

July 29 - August 3, 2013

Mainz, Germany

\footnotetext{
* Speaker.
} 


\section{Introduction}

The discovery of the Higgs particle this year has greatly increased the effort of the lattice field theory community in exploring models of the Higgs mechanism of spontaneous symmetry break in a non-perturbative setting. It is widely accepted that the physics represented by the scalar Higgs field within the electroweak sector of the Standard Model is an effective theory of higher energy phenomena.

One such approach is to model the Higgs particle as a scalar bound state arising from constituent "techni"-fermions interacting via a non-abelian "technicolor" gauge field, modelled on QCD, and many talks at this conference have been devoted to finding the appropriate gauge symmetry and fermion content of that model. Another approach to achieving spontaneous symmetry breaking is via the Hosotani Mechanism [1],[2], [3]. In this scenario, one introduces compactified extra spatial dimensions; the Higgs field is then a manifestation of the zero-mode of the "normal" gauge field in the theory.

\section{The Hosotani Mechanism}

The Hosotani Mechanism works in a space with at least one compactified dimension, which is typically taken to be four-dimensional Minkowsky spacetime crossed with a circle: $M^{3,1} \times S^{1}$. On the lattice, we will use a Euclidian torus, and simply have one dimension small compared to the others. In calculations of what situations give rise to the Hosotani Mechanism, there is nothing special about the number of uncompactified dimensions (it is a parameter in the effective potential), so for this study we take that number to be three. This choice makes lattice simulations much more staightforward.

Clearly this is very similar to a finite temperature scenario, and it is important to understand the differences and similarities between high temperature physics and the Hosotani Mechanism. The gauge field is parameterized as $A_{\mu}(x, y)$, with $y$ the coordinate of the compact dimension. When the size of the compact dimension is small, the Matsubara frequencies of the $A_{4}$ field are well separated and only the lowest-the zero mode-is relevant. Thus $A_{4}(x, y)=A_{4}(x)$ and essentially becomes an scalar field in the uncompactified space. The dynamics and vacuum expectation value of this scalar field are determined by the effective potential for $A_{4}, V_{\text {eff }}\left(A_{4}\right)$, which in turn is determined by the fermion (and other field) content of the theory. In certain cases, particularly with adjoint fermions having periodic boundary conditions, the $A_{4}$ field develops a VEV which lies outside the center of the gauge group, and the expectation is that the gauge symmetry is broken. Testing this scenario non-perturbatively is the purpose of this study, as well as others at this conference [4] and [5].

For $\mathrm{SU}(\mathrm{N})$, the zero-mode of the $A_{4}$ field is usually parameterized as

$$
\left\langle A_{y}\right\rangle=\frac{1}{g L}\left(\begin{array}{ccc}
\theta_{1} & & \\
& \ddots & \\
& & \theta_{N}
\end{array}\right), \quad \sum_{i=1}^{N} \theta_{i}=0
$$

The calculation of the effective potential is standard [1].

$$
V_{\mathrm{eff}}=\sum( \pm) \frac{i}{2} \operatorname{tr} \ln D^{M} D_{M}
$$


where

$$
D^{M} D_{M}=\partial^{\mu} \partial_{\mu}-D_{y}^{2}\left(\theta_{i}\right)
$$

We take the sign + or - for bosons or fermions, respectfully, yielding

$$
V_{\mathrm{eff}}(\boldsymbol{\theta})=\sum( \pm) \frac{1}{2} \int \frac{d^{d} p}{(2 \pi)^{d}} \sum_{n} \ln \left\{p^{2}+\frac{1}{R^{2}}\left(n+\left(\frac{\theta}{2 \pi}\right)^{2}\right\}\right.
$$

The sums take the following forms for the gauge and ghost contributions, fermions in the fundamental representation, and fermions in the adjoint representation.

$$
\begin{aligned}
V_{\mathrm{eff}} & =V_{\mathrm{eff}}^{A_{\mu}}+V_{\mathrm{eff}}^{\psi}, \quad \theta_{N}=\sum_{i=1}^{N-1}-\theta_{i}, \\
V_{\mathrm{eff}}^{A_{\mu}} & =-(d-2) \frac{\Gamma(d / 2)}{\pi^{d / 2} L^{d}} \sum_{i, j=1}^{N} \sum_{n=1}^{\infty} \frac{\cos \left[n\left(\theta_{i}-\theta_{j}\right)\right]}{n^{d}}, \\
V_{\mathrm{eff}}^{\psi, \mathrm{f}} & =N_{\mathrm{f}} \cdot 2^{[d / 2]} \frac{\Gamma(d / 2)}{\pi^{d / 2} L^{d}} \sum_{i=1}^{N} \sum_{n=1}^{\infty} \frac{\cos \left[n\left(\theta_{i}-\beta_{\mathrm{f}}\right)\right]}{n^{d}}, \\
V_{\mathrm{eff}}^{\psi, \mathrm{ad}} & =N_{\mathrm{ad}} \cdot 2^{[d / 2]} \frac{\Gamma(d / 2)}{\pi^{d / 2} L^{d}} \sum_{i, j=1}^{N} \sum_{n=1}^{\infty} \frac{\cos \left[n\left(\theta_{i}-\theta_{j}-\beta_{\mathrm{ad}}\right)\right]}{n^{d}} .
\end{aligned}
$$

The $\beta$ 's represent the boundary conditions (B.C.s) on the fields: $\psi(y+2 \pi R)=e^{i \beta} \psi(y) . d$ is the dimension of the spacetime.

The complete effective potential $V_{\text {eff }}\left(\theta_{i}\right)$ is the sum of the appropriate functions above. For fermions in the fundamental representation, any B.C. we choose leads to a the minimum of the effective potential in which the VEV for $\left\langle A_{4}\right\rangle=\operatorname{diag}\left(\theta_{1}, \theta_{2}, \theta_{3}\right)$ lies in the center of SU(3), and hence the gauge symmetry is not broken. However, for fermions in the adjoint representation we see that the potential functions have similar periods in $\theta_{1}$ and $\theta_{2}$, but have opposite signs. This allows for interference between the gauge and fermion contributions to $V_{\text {eff }}$ and non-trivial minima in which the VEV of $\left\langle A_{4}\right\rangle$ lies outside the center of SU(3).

The possible cases are nicely summarized in [6]. There, four phases are defined as X, A, B, and C. Additionally, this model-SU(3) gauge theory with 2 flavors of staggered fermions in four dimensions with one direction much smaller than the others-was studied by Cossu and D'Elia in [7]. Those authors found new phases as they made the radius of the compact dimension smaller and smaller, which they called confined, deconfied, split, and reconfined. Table 1. below (taken from [6]) summarizes these phases, the VEVs for $A_{4}=\operatorname{diag}\left(\theta_{1}, \theta_{2}, \theta_{3}\right)$, the values of the Polyakov loops in the fundamental and adjoint representations, and the symmetry that commutes with the Polyakov loop in the compact dimension.

The simulations in [6] and [7] confirm these results non-perturbatively, in that they show the expectation values listed above for the Polyakov loops in each phase. The distributions of the Polyakov loop values are also consistent with the effective potential forms shown above. The next step is to investigate the nature of the local gauge symmetry breaking (if any) that occurs in the different phases. 


\begin{tabular}{|c|c|c|c|c|}
\hline Phase name: [6], [7] & $\left\langle\left(\theta_{1}, \theta_{2}, \theta_{3}\right)\right\rangle+$ perms., $\bmod 2 \pi$ & $P_{3}$ & $P_{8}$ & Symmetry \\
\hline$X$, Confined & Large quantum fluctuations & 0 & $-\frac{1}{8}$ & $\mathrm{SU}(3)$ \\
\hline$A_{1,2,3}$, Deconfined & $(0,0,0),\left( \pm \frac{2}{3} \pi, \pm \frac{2}{3} \pi, \pm \frac{2}{3} \pi\right)$ & $1, e^{ \pm 2 \pi i / 3}$ & 1 & $\mathrm{SU}(3)$ \\
\hline$B_{1,2,3}$, Split & $(0, \pi, \pi),\left( \pm \frac{2}{3} \pi, \mp \frac{1}{3} \pi, \mp \frac{1}{3} \pi\right)$ & $-\frac{1}{3}, \frac{1}{3} e^{\mp \pi i / 3}$ & 0 & $\mathrm{SU}(2) \times \mathrm{U}(1)$ \\
\hline$C$, Reconfined & $\left(0, \frac{2}{3} \pi,-\frac{2}{3} \pi\right)$ & 0 & $-\frac{1}{8}$ & $\mathrm{U}(1) \times \mathrm{U}(1)$ \\
\hline
\end{tabular}

Table 1: Phases of 4-dim. SU(3) gauge theory with two flavors of adjoint fermions, showing the locations of the global minima of $V_{\text {eff }}\left(\theta_{i}\right) . P_{3}$ and $P_{8}$ are the fundamental and adjoint Polyakov loop in the 4-direction.

\section{Gauge Symmetry Breaking}

To recap, as a simpler example of the Hosotani Mechanism in 4+1 dimensions, we study a $3+1$ dimensional gauge theory with two staggered fermions having periodic B.C.s, and adjust the gauge coupling $\beta$ to vary the radius of the compact dimension. This situation is very similar to the extensively studied case of gauge theories at finite temperature. In the finite temperature case, the Polyakov loop also looses a global symmetry, in that case $Z_{3}$. Thus it is important to understand the differences between the deconfinement transition in finite temperature gauge theory and the Hosotani Mechanism.

One of the most important features of dynamical symmetry breaking via the Higgs mechanism is the appearance of massive gauge bosons, which transforms the physics of the theory significantly. Thus we first look for indications of gauge symmetry breaking in the gluon propagator.

The lattices studied are listed below, and were generously provided for study by E. Itou and her collaborators. They contain two flavors of staggered fermions. The standard Wilson action was used for the gauge field and Kogut-Susskind action for the adjoint fermions.

\begin{tabular}{|c|c|c|c|}
\hline Phase & $\beta$ & dimensions & fermion mass \\
\hline Confined & 5.30 & $16^{3} \times 4$ & 0.1 \\
\hline Deconfined & 5.70 & $16^{3} \times 4$ & 0.1 \\
\hline Split & 5.95 & $16^{3} \times 4$ & 0.1 \\
\hline Reconfined & 5.30 & $16^{3} \times 4$ & 0.1 \\
\hline
\end{tabular}

Table 2: Lattice ensembles used in this study, provided by E. Itou and collaborators

To check whether the gauge boson develops a mass, we compute the gluon propagator. We define

$$
A_{\mu}(x)=\frac{1}{2 i a}\left[U_{\mu}(x)-U_{\mu}^{\dagger}(x)\right]_{\text {Traceless }}
$$

and fix the gauge to Landau gauge by iteratively maximizing the functional

$$
I\left(U_{\mu}, G\right)=\operatorname{Re} \operatorname{Tr} \sum_{x} G^{\dagger}(x) U_{\mu}(x) G(x+\mu)
$$




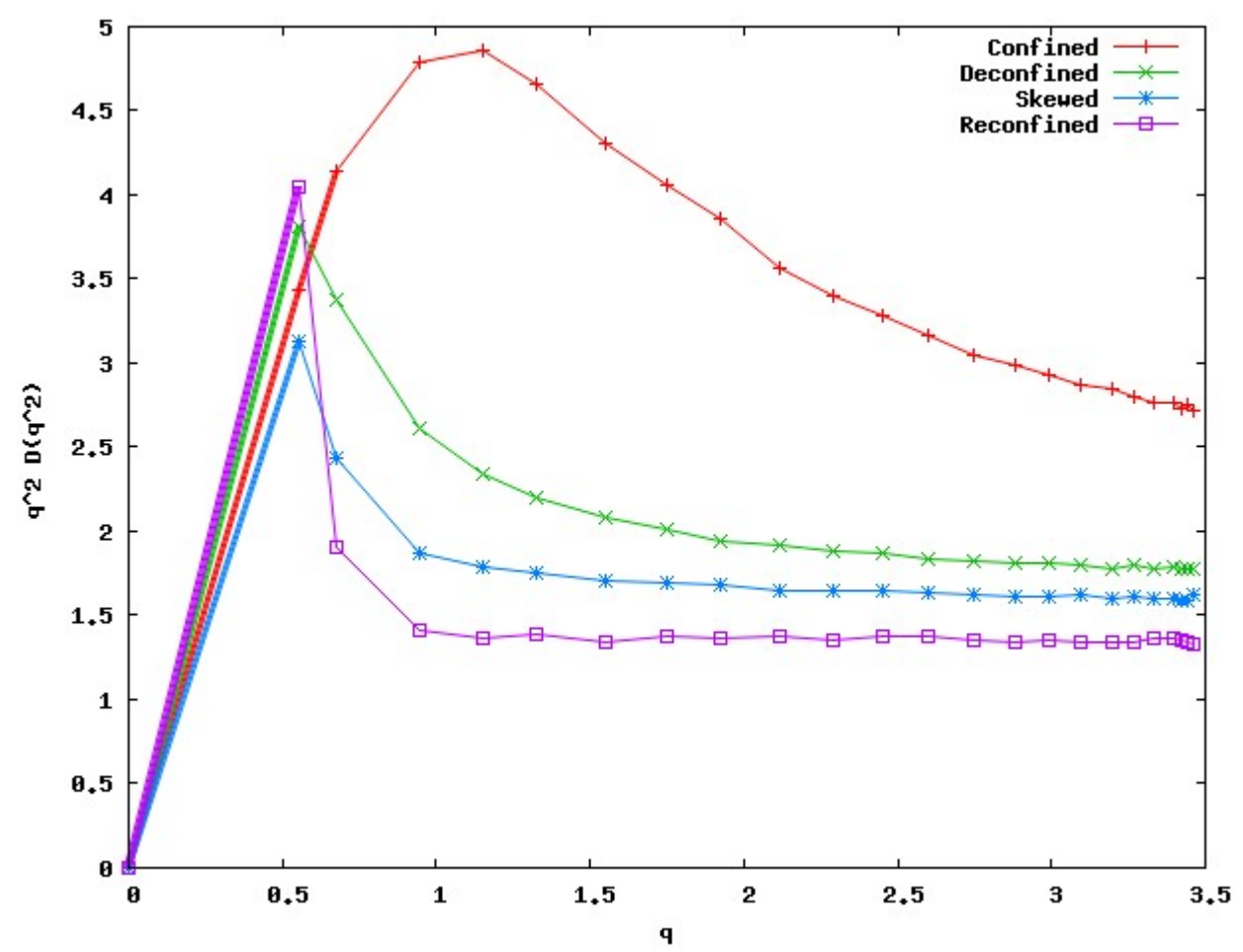

Figure 1: The gluon propagator in Landau gauge, measured on the ensembles and phases listed in Table 2.

with respect to $G(x)$. The gluon propagator, defined in momentum space is:

$$
<\hat{A}_{\mu}^{a}(q) \hat{A}_{v}^{b}\left(q^{\prime}\right)>\equiv \mathscr{D}_{\mu v}^{a b}(q)=\left(\delta_{\mu v}-\frac{q_{\mu} q_{v}}{q^{2}}\right) \delta^{a b} D\left(q^{2}\right)
$$

Figure 1 shows the average gluon propagator measured on each of the ensembles in Table 2. The $q^{2}$-weighted scalar part of the propagator, $q^{2} D\left(q^{2}\right)$, is plotted, which is a standard way to plot the gluon propagator (see for example, [8]).

What we find is a significant change in the propagator from the Confined to Deconfined phase, but little change in the propagator as we move to the other broken-symmetry phases, split $[\mathrm{SU}(2) \times \mathrm{U}(1)]$ and reconfined $[\mathrm{U}(1) \times \mathrm{U}(1)]$. The flat line indicates that $D\left(q^{2}\right)$ is proportional to $1 / q^{2}$, i.e. massless. Further, the behavior of the propagators in the "broken-symmetry" phases, Split and Reconfined corresponding to the B and C phases in Table 1, show little change from the SU(3) symmetric Deconfined (A) phase.

Another test was to compute the spatial correlator of time-like Polyakov loops. The decay of this correlator is related to the glueball mass among other things, and one might hope to see some indication of dynamical symmetry breaking in these correlators. As before, we compute them and look for different qualitative behavior between the deconfined, split, and reconfined phases. The quantity of interest is

$$
c(r)=\operatorname{Re}<\operatorname{Tr} P(x) \operatorname{Tr} P^{\dagger}(x+r)>-<\operatorname{Tr} P \operatorname{Tr} P^{\dagger}>
$$

and $c(r)$ is plotted in Figure 2 for the ensembles in Table 2. 


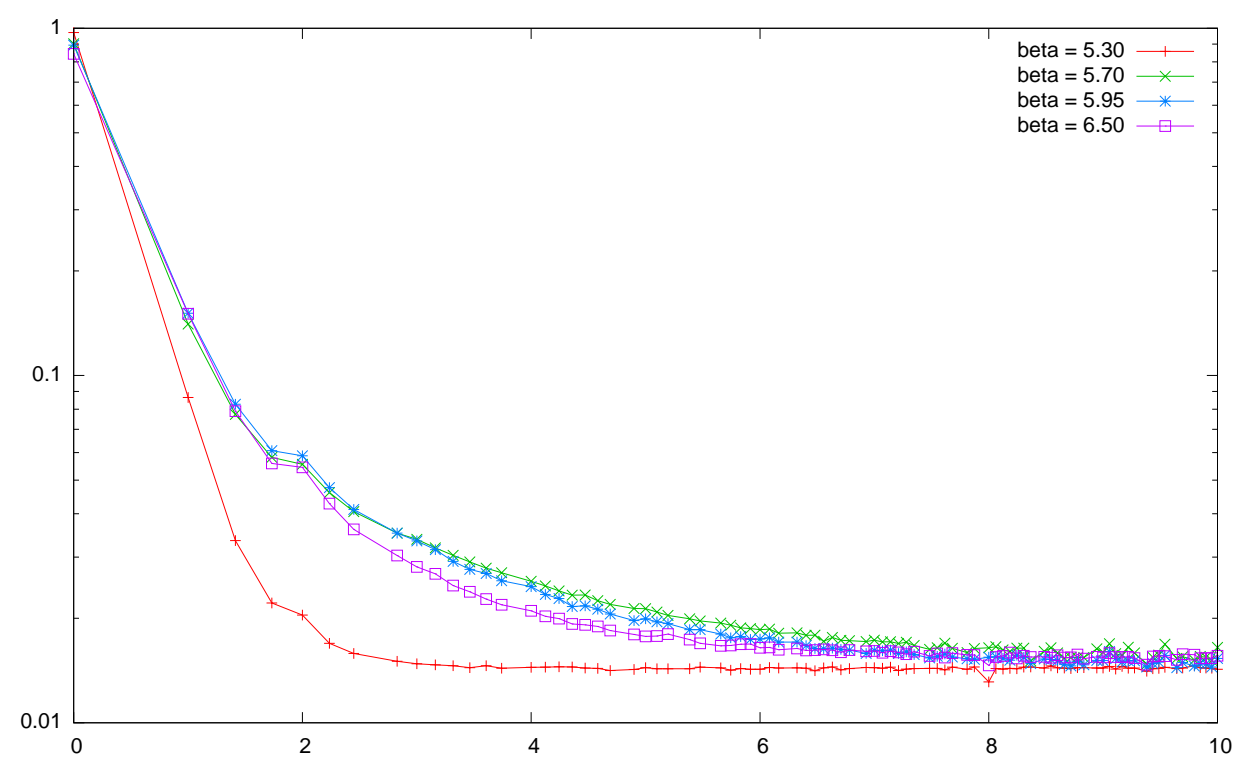

Figure 2: The spatial correlator of Polyakov loops wrapped around the compact dimension

Again, we see a clear difference in behavior between the Confined and other phases, but little difference between the Deconfined (A), Split (B), and Reconfined (C) phases.

As a final test, we computed the spatial correlator of elements of the time-like (compact direction) Polyakov loop matrix elements among themselves.

$$
c_{i j m n}(r)=<P_{i j}(x) P_{m n}^{\dagger}(x+r)>
$$

While these elements are gauge dependent, the hope is that if the gauge symmetry is broken, the nature of the correlations would change amoung some subset of correlators. An example of our results are shown in Figure 3, for the ensemble in the Split $[\mathrm{SU}(2) \times \mathrm{U}(1)]$ phase. What we see is that the diagonal correlators $\left\langle\sum_{x} p_{i j}(x) p_{m n}(x+r)\right\rangle$ in which $i j m n=0011,1122$, or 0022 , are nonzero, whereas all others are very small. This behavior persists on all ensembles-only the diagonal elements have significant correlations between themselves.

\section{Conclusions}

It is encouraging that several groups within the lattice community are starting to explore the Hosotani Mechanism non-perturbatively. The results of [5] show very good agreement between the calculations of the effective potential $V_{\text {eff }}\left(\theta_{i}\right)$ and the results of simulation, in which this potential is extracted. The Polyakov loop in the cases studied indeed breaks the global symmetries as shown in Table 1.

On the other hand, the local gauge field properties as displayed by the gluon propagator and the $P(x) P^{\dagger}(x)$ correlators in the Deconfined [SU(3) symmetric], Split [SU(2) $\left.\times \mathrm{U}(1)\right]$, and Reconfined $[\mathrm{U}(1) \times \mathrm{U}(1)]$ phases shows very little qualitative difference. 


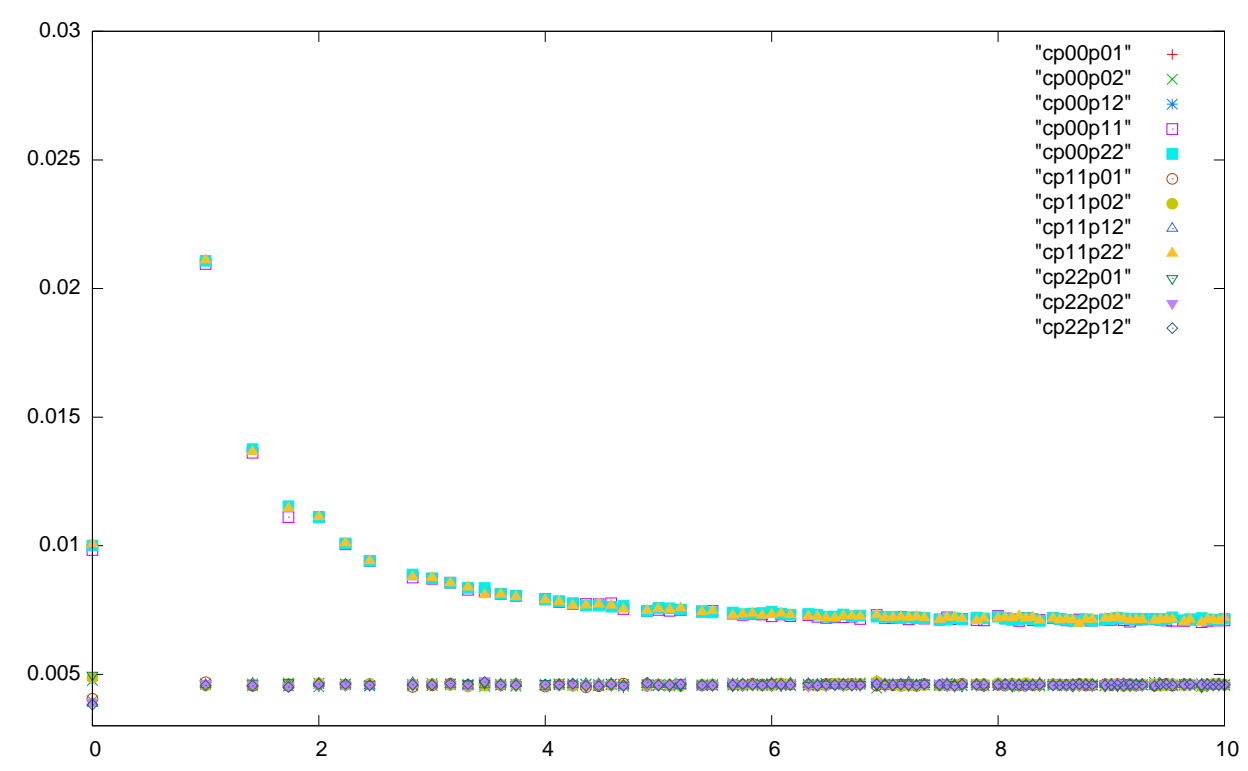

Figure 3: The spatial correlator of Polyakov loop elements in the Split $\mathrm{SU}(2) \times \mathrm{U}(1)$ phase.

It is quite possible that the behavior of the $3+1$ model studied here (and in refs. [6] and [7] are significantly different with respect to dynamical gauge symmetry breaking than what is expected in the 4+1 dimensional model outlined in Hosotani's original paper [1].

One interesting line of research to follow this work would be to explicitly write out the dimensionally reduced 3 - and 4-dimensional models, including the effective potential, $V_{\text {eff }}$, produced by the adjoint fermions, and see what differences occur.

\section{References}

[1] Y. Hosotani, Phys. Lett. B 126, 309 (1983).

[2] Y. Hosotani, Phys. Lett. B 129, 193 (1983).

[3] J. E. Hetrick and C. -L. Ho, Phys. Rev. D 40, 4085 (1989).

[4] K. Kashiwa, LATTICE 2013 (This Proceedings), and, K. Kashiwa and T. Misumi, "Phase structure and Hosotani mechanism in gauge theories with compact dimensions revisited," JHEP 1305, 042 (2013) [arXiv:1302.2196 [hep-ph]].

[5] G. Cossu, E. Itou, H. Hatanaka, Y. Hosotani and J. -I. Noaki, LATTICE 2013 (This Proceedings) "Hosotani mechanism on the lattice," arXiv:1311.0079 [hep-lat].

[6] G. Cossu, H. Hatanaka, Y. Hosotani and J. -I. Noaki, "Polyakov loops and the Hosotani mechanism on the lattice," arXiv:1309.4198 [hep-lat].

[7] G. Cossu and M. D’Elia, JHEP 0907, 048 (2009) [arXiv:0904.1353 [hep-lat]].

[8] C. Alexandrou, P. de Forcrand and E. Follana, Phys. Rev. D 63, 094504 (2001) [hep-lat/0008012]. 